University of Wollongong

Research Online

Faculty of Engineering and Information

Faculty of Engineering and Information

Sciences - Papers: Part A

Sciences

$1-1-2014$

Electro-tactile feedback system for achieving embodiment in a teleoperated robot

Daniel Pamungkas

University of Wollongong, dsp572@uowmail.edu.au

Koren Ward

University of Wollongong, koren@uow.edu.au

Follow this and additional works at: https://ro.uow.edu.au/eispapers

Part of the Engineering Commons, and the Science and Technology Studies Commons

Research Online is the open access institutional repository for the University of Wollongong. For further information contact the UOW Library: research-pubs@uow.edu.au 


\title{
Electro-tactile feedback system for achieving embodiment in a tele-operated robot
}

\begin{abstract}
Tele-operation can enable an operator to control a robot remotely in inaccessible and hazardous environments. However, controlling a robot remotely via a conventional monitor and control panel can be difficult and slow. To achieve faster and more dexterous operation of the robot, enhanced 3D perception and some form of tactile or neural feedback is needed to achieve some degree of embodiment within the robot's physical structure and world. To achieve this objective we have devised an immersive teleoperation system comprised of a stereo vision headset and an electro-tactile feedback system that is worn by the operator, connected to stereo cameras and various sensors mounded on the robot. This arrangement enables the remote operator to see in 3D what the robot sees and experience what the robot feels via electro-tactile feedback in response to hand gesture based control actions. We provide experimental results showing how our tele-operation system can enable the operator to achieve better control of a mobile robot via a sense of being partially "embodied" within the robot.
\end{abstract}

\section{Keywords}

electro, tele, embodiment, achieving, system, robot, feedback, operated, tactile

\section{Disciplines}

Engineering | Science and Technology Studies

\section{Publication Details}

Pamungkas, D. S. \& Ward, K. (2014). Electro-tactile feedback system for achieving embodiment in a teleoperated robot. 13th International Conference on Control Automation, Robotics and Vision (pp. 1706-1711). United States: Institute of Electrical and Electronics Engineers. 


\section{Electro-tactile Feedback System for Achieving Embodiment in a Tele-Operated Robot}

\author{
Daniel S Pamungkas \\ School of Computer Science and Software Engineering \\ University of Wollongong \\ Wollongong, Australia \\ dsp572@uowmail.edu.au
}

\author{
Koren Ward \\ School of Computer Science and Software Engineering \\ University of Wollongong \\ Wollongong, Australia \\ koren@uow.edu.au
}

\begin{abstract}
Tele-operation can enable an operator to control a robot remotely in inaccessible and hazardous environments. However, controlling a robot remotely via a conventional monitor and control panel can be difficult and slow. To achieve faster and more dexterous operation of the robot, enhanced 3D perception and some form of tactile or neural feedback is needed to achieve some degree of embodiment within the robot's physical structure and world. To achieve this objective we have devised an immersive tele-operation system comprised of a stereo vision headset and an electro-tactile feedback system that is worn by the operator, connected to stereo cameras and various sensors mounded on the robot. This arrangement enables the remote operator to see in 3D what the robot sees and experience what the robot feels via electro-tactile feedback in response to hand gesture based control actions. We provide experimental results showing how our tele-operation system can enable the operator to achieve better control of a mobile robot via a sense of being partially "embodied" within the robot.
\end{abstract}

Keywords - Embodiment, electro-tactile feedback, TeleOperation

\section{INTRODUCTION}

Tele-operated robot technologies can allow humans to work in hazardous or remote places from a safe location. For example, an operator can control robotic equipment at distant or inhospitable locations conveniently from their office, greatly reducing both the operator's travel time and injury risk [1]. Such systems can also allow the operators interactions with the environment to be scaled up or down to achieve larger or smaller action and forces. There are many applications of tele-operation such as: mining machinery [2], underwater maintenance [3], bomb disposal [4], hazardous waste removal [5], space missions [6] and tele-surgery [7].

Dolezal [8] states that tele-operation can be successful if there is a good match between the perception and interactions of the operator and the robot. Furthermore, Such a close coupling between perception and action is an important factor is in achieving a sense of the embodiment with the robot as a consequences of tele-operation as explained by Haans and Ijsselsteijn [9]. Blanke and Metzinger [10] state that embodiment can be the experience and effect of having and using a body in a virtual environment or virtual agent within the robot. [11].
Embodiment only happens when the properties of the agent/robot are processed similarly to the properties of the real body [12]. To achieve embodiment, three sense concepts must be incorporated, namely: sense of location, sense of agency and sense of body ownership. Sense of location refers to the out of body experience in which the operator perceives themselves to be outside their real body [13]. Sense of agency refer to the sense of having control [10]. Finally, sense of body ownership relates to body feeling sensations [14]. To fulfill these requirements a tele-operation system must have an appropriate operator interface to both control the robot and achieve multi sensory feedback. Most existing tele-operation systems that attempt to fulfill these requirements are cumbersome and complex making it difficult for an operator to achieve a sense of embodiment with the robot.

This paper proposes a combined 3D perception and electro-tactile feedback system to address some of these drawbacks. Our system utilizes a data glove to control the robot, a 3D stereo-vision headset to perceive the robot's environment and multiple channels of tactile feedback achieved with wireless electro-tactile feedback units.

3D stereo-vision helps the operator to gain depth perception that is not available with conventional display screens [15]. In addition, the electro- tactile feedback which delivers a mild electric current to specific nerves within the skin which can enrich the operator's awareness of the robot's situation and interactions with environment [16].

This paper is organized as follows: In Section II we provide a brief overview of previous research on visual and haptic feedback systems with respect to controlling robots via tele-operation. In Section III the implementation details of our tele-operation system are presented. Section IV describes experiments we undertook which demonstrate the effectiveness of our tele-operation system. Concluding remarks are provided in Section V. 


\section{BACKGROUND}

One of the first classical experiments performed to demonstrate the concept of embodiment is called the Rubber Hand Illusion (RHI) [17]. This involves placing a substitute rubber hand in a position where it appears to be one of the subject's own hands and subjecting it to the same sensations as the real hand which is placed out of view of the subject. When the user reports that the rubber hand feels like it is their own hand the demonstrator then impacts the rubber hand with a mallet with usually makes the subject exhibit considerable distress.

Although the RHI experiment demonstrates the concept of embodiment, achieving the same effect with robotic equipment has proven to be a more challenging task. This usually involves devising a human-computer interface that enables the robot to be remotely controlled at the operator's will while simultaneously seeing what the robot sees and experiencing what the robot feels. If done well the operator feels as if he is physically transposed into the robot's body and capable of interacting with the environment dexterously. However, most existing immersive tele-operation systems fall somewhat short of this goal.

Tele-operation can be established by using a joystick to control a robot with video on screens as feedback [18], [19]. Although such systems can give the operator adequate control of the robot and sufficient perception of the robot's world, this arrangement is generally not sufficient to achieve a sense of embodiment. VR headsets can help to make the operator feel more immersed in the robot's world, however headsets which use monoscopic vision may have difficulties where the task requires depth perception [15], [20].

To achieve a more immersive experience and improved depth perception stereoscopic vision is needed. Stereoscopic head mound display can also increase the agility and efficiency of the operator [21] especially if some degree of embodiment is achieved. However, interacting with the environment can still be difficult if no tactile feedback is provided.

To improve interactivity haptic feedback can be added to the tele-operator system. This usually involves devising some type of feedback systems which can be felt via the operator's skin, for example: heat [22], vibration [23], force [24] and electricity [25]. Visual and haptic feedback can also improve the performance of the tele-operation system and decrease control effort needed to perform specific tele-operation tasks. For example, in [7] a tele-operation system with haptic feedback was used to facilitate tele-surgery with positive results. This system made the operator more aware of the pressure of tele-operated surgical instruments. In [26], experiments were performed to determine how to control a robot doing various assembly tasks, both with and without haptic feedback. Similar experiments were also conducted in virtual reality to determine how a haptic feedback system can improve the interactivity and speed of various assembly tasks [27].

However, equipping both the robot and the operator with a haptic feedback system can be difficult to setup, complex and expensive, particularly if the feedback is delivered to the operator via electro-mechanical linkages. For example, in [24] feedback information on the position of a robot arm is delivered to the operator via pulleys attached to weights to effect forces between the operator's waist and hand. In [28], feedback sensations such as tapper, dragger, squeezer and twister are achieved using servo motors.

Vibration has also been used to deliver haptic feedback for performing various tasks. in [23], a vibro-tactile and mechanical torque feedback system was used for controlling a robotic arm. Here, the operator's arm connected by a belt to a DC motor. This system is used as force feedback to link the robot arm to the operator. Information about the profile of objects surrounding the robot's gripper via six vibro-tactile feedbacks implemented as servo actuated bracelets.

As all these haptic feedback systems involve electromechanical actuators and/or linkages, they are rather cumbersome to wear and can confine the operator's motions. Moreover, they can make it difficult for the operator to achieve embodiment because the operator becomes more aware of the feedback mechanisms attach to the operator's body than the robot's environment, as explained in [29].

An electro-tactile feedback device produces varying electric current to stimulate nerves within the skin via electrodes in order to deliver haptic feedback to the user. Consequently, this form of haptic feedback system can give a variety of sensations without the need for mechanical actuators or linkages. This can dramatically reduce the amount of hardware needed to deliver haptic feedback to the operator.

Another advantage of electro-tactile feedback is that it can be modulated by varying the frequency and amplitude of the voltage delivered through the skin to the sensory nerves. This can give a diverse range of sensations, without desensitizing the nerves as explained in [16], [25] and [30].

Although electro-tactile feedback cannot replicate all the tactile sensations experienced by humans via the sense of touch, it can deliver analogues that can enable the user to experience a wide range of sensations from a variety of sensing devices and respond appropriately. In the following sections we describe the implementation details of our electrotactile tele-operation system and some preliminary experiments that we were able to achieve.

\section{ELECTRO-TACTILE FEEDBACK SYSTEMS}

To demonstrate the benefits of our electro-tactile teleoperation system we equipped a mobile robot with stereo cameras, distance sensors and a gripper, as shown in Figure 1. The robot is also equipped with a pressure switch for detecting when an object is gripped by the robot and odometry which is used for facilitating the detection of objects over time by use of an occupancy grid. 


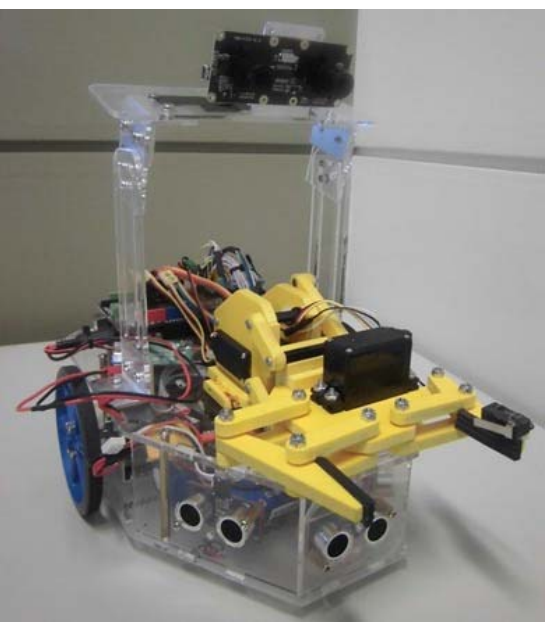

Figure 1. Mobile robot.

Information from the stereo cameras and sensors is then transmitted wirelessly to the operator's computer and then to the stereo headset and custom built electro-tactile data glove, as shown in Figure 2. The stereo headset enables the operator to view the immediate environment in front of the robot via the stereo cameras in $3 \mathrm{D}$ which give the operator the impression that his eyes are positioned on the robot's turret.

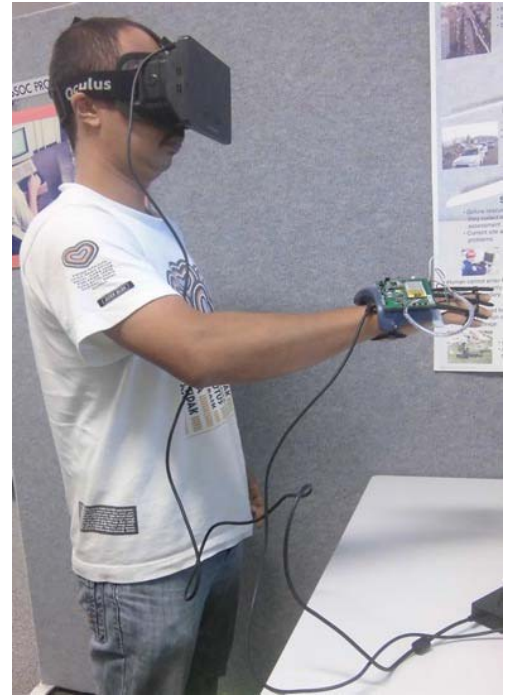

Figure 2. Operator with data glove and the feedback system.

Information from the robot's range sensors and gripper pressure switch is delivered to the electro-tactile data glove which has TENS electrodes fitted and arranged to enable electro-stimulus to be delivered to the operator's fingers. As the latency of both electro-tactile feedback system and visual feedback is less than $200 \mathrm{~ms}$ no synchronization measures were needed.

The data glove is also equipped with a tracking system and finger bend sensors which enables this device to map hand gestures to control commands delivered to the robot. This control-feedback system is intended to make the operator's hand feel as if it is "gloved" or "immersed" into the mobile robot and capable "feeling" sensor stimulus from the robot's local environment and directing the robot's motion in an intuitive manner. Figure 3 shows a basic overview of the electro-tactile tele-operation systems and the feedback loop. Further implementation details of the robot and control feedback system are provided in the following sections.

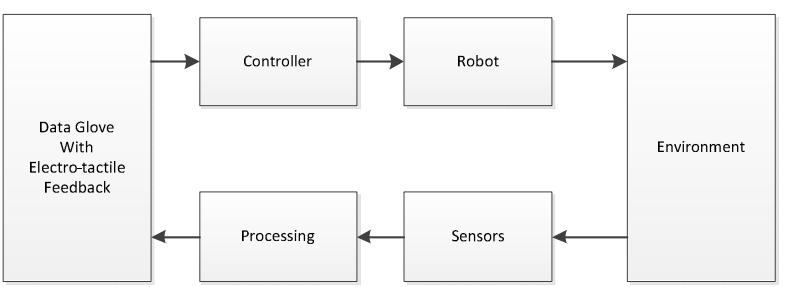

Figure 3. Block diagram of electro-tactile tele-operation system.

The implementation details of the control, feedback system, and the robot are provided in the following sections

\section{A. Mobile Robot}

The mobile robot measures approximately $25 \mathrm{~cm} \times 18 \mathrm{~cm}$ $\mathrm{x} 25 \mathrm{~cm}(\mathrm{~L} \times \mathrm{W} \times \mathrm{H})$ and is shown in Figure 1. It has two drive wheels, one idler wheel and a gripper that is suitable for picking up a drink can. The robot has a maximum speed of 20 $\mathrm{cm} / \mathrm{s}$ in the forward and reverse directions and is equipped with an xbee wireless modem for communications between its main processor and the host computer.

The robot is also equipped with Ovrvision stereo camera fitted to the robot's turret for providing the user with 3D forward vision of the robot's environment, as shown in figure 1. The Ovrvision stereo cameras operate at $60 \mathrm{fps}$ with low latency and have $1280 \times 480$ pixel resolution. The stereo cameras are connected an Oculus Rift head-mounted display.

Four ultrasonic distance sensors and a pressure switch are used for sensing obstacles in the environment as well as the presence or absence of an object in the gripper. The ultrasonic range sensors are mounted on the body of the mobile robot and are used for detecting objects in the near vicinity of the robot. One sensor is facing the forward direction (or $0^{0}$ ). Two sensors are aimed right and left $\left(45^{\circ}\right.$ and $\left.-45^{\circ}\right)$ of the robot. Another distance sensor is facing the reverse direction $\left(180^{\circ}\right)$ of the robot. For this experiment, we set the maximum range of the ultrasonic sensors to $60 \mathrm{~cm}$.

\section{B. Data Glove}

To control the movement of the robot, a P5 Virtual Reality Glove is used, as shown in Figure 4 and 5. This device can give the coordinates of the glove's $\mathrm{x}, \mathrm{y}, \mathrm{z}$ position as well as the glove orientation in the terms of roll, pitch and yaw. The bend positions of all fingers are also available. Additional signals from this glove can be delivered by three buttons mounted on the back shell. The glove is placed in front of its receptor tower to monitor and read the glove's position and state, as shown in Figure 4. To integrate with the electrotactile feedback, a custom built five channel electro-tactile feedback receptor unit is attached to the glove's back shell, see Figure 4. Each output from this receptor is delivered to electrodes mounted on the bend sensors, as shown in Figure 5. 
The TENS electrodes are coded with conductive gel to establish electrical contact with the back of the fingers.

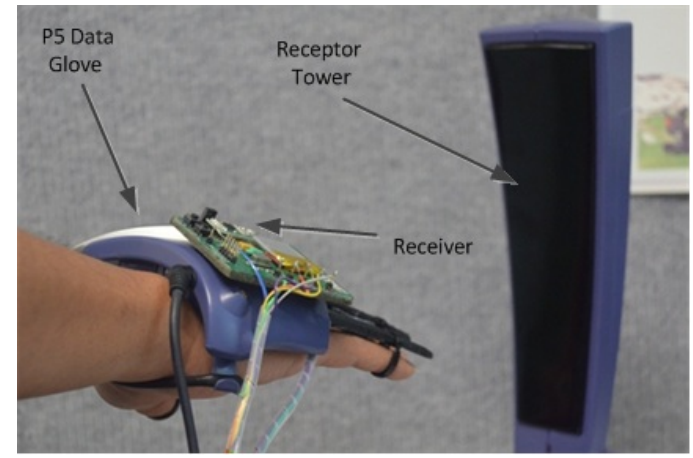

Figure 4. Data Glove with feedback unit and receptor tower.

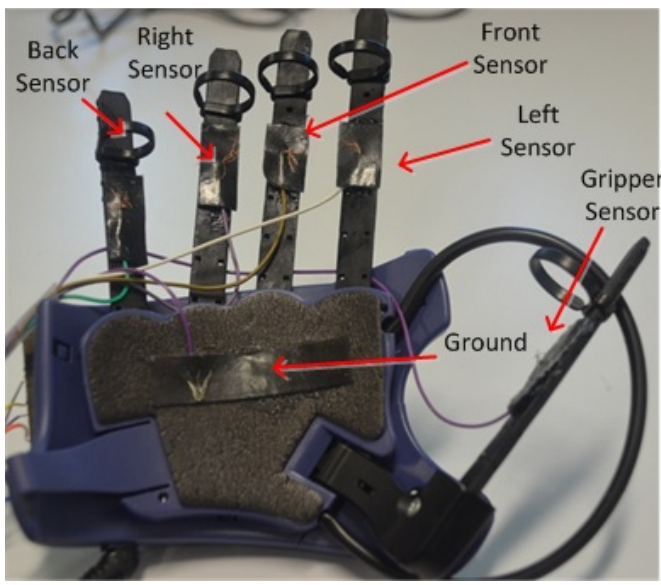

Figure 5. TENS electrodes fitted to data glove.

To control the robot with the data glove hand gestures are used. To move the robot in the forward or reverse direction the pitch of the glove is used. To move the robot in the left or right direction the glove's roll position is used. Bending the fingers up or down slightly raises or lowers the gripper. Bending the thumb opens or closes the gripper. This protocol was found to be sufficiently intuitive for the operator to be able to control a robot within its workspace and relocate cans without much practice being required.

\section{Electro Tactile Feedback}

To deliver tactile feedback from the robot's range sensors to the operator's hand, a custom built five channels wireless TENS system is used, as shown in Figure 6. This system can provide up to five channels of electrical stimulus to the operator's skin with both controlled frequency and intensity. The electro tactile feedback system consists of a USB transmitter, shown in Figure $6 \mathrm{a}$, and the receiver unit shown in Figure $6 \mathrm{~b}$. The transmitter unit transmits the feedback data from the robot's micro controller which is derived from the robot's sensors. The receiver unit receives this information wirelessly and converts this data into varying electrical pulses delivered to fingers, as shown on Figure 7 and Figure 8.

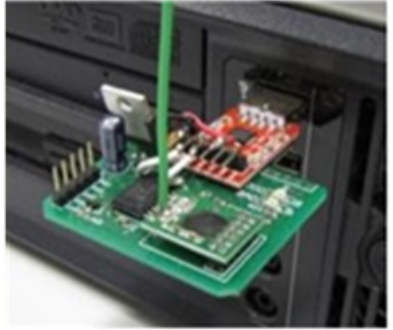

a

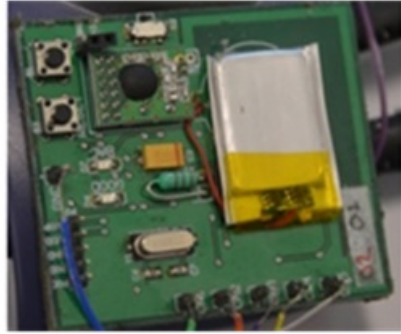

Figure 6. a.USB transmitter b.TENS receiver unit.

The feedback electrodes are fitted to the inside surface of data glove so that they make contact with the back of the operator's fingers. Also, the ground electrode is placed in the center of the glove to make contact with the back of the hand. The electrode positions and tactile feedback protocol is illustrated in Figure 7. This arrangement allows the operator to receive five channels of stimulus information via the fingers, as explained in the previous section. The stimulus does not cause the hand to contract because the electrodes are not directly stimulating to the mussels. In fact, the stimulus is mild, completely painless and does not affect use of the hand.

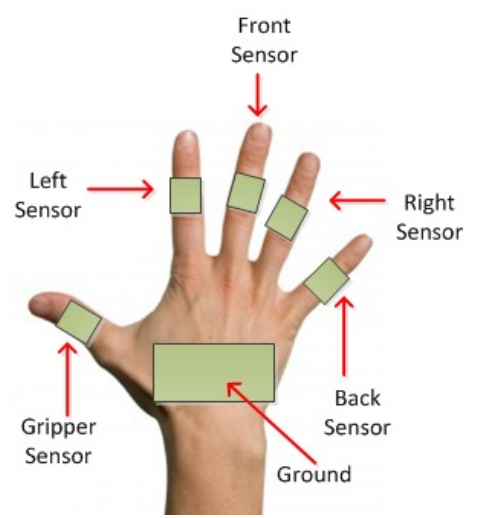

Figure 7. Position of the feedback electrodes.

The stimulus pulses have a frequency at $20 \mathrm{~Hz}$, as shown in Figure 8 . The amplitude of the pulses are set by the operator between $40 \mathrm{~V}$ to $80 \mathrm{~V}$ to suit the operator's comfort level. Intensity is controlled by the width of the pulse and the pulse width of the signal is varied between 10 to $100 \mu \mathrm{s}$, depending on the signal from the robot's sensors.

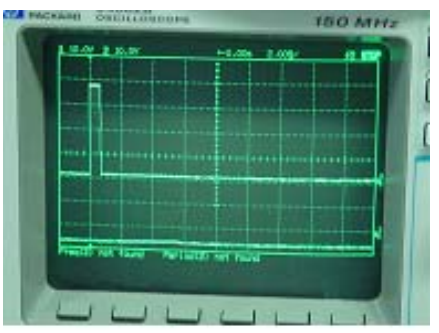

Figure 8. TENS output waveform. 


\section{Stereo vision}

The vision feedback system consists of stereo camera from OVR which delivers stereo images to the Oculus Rift headmounted display unit worn by the operator, as shown in Figure 9. This equipment provides the user with $3 \mathrm{D}$ stereoscopic vision with 1000 field of view, as shown in Figure 9. The latency is also relatively fast enabling operator to see the robot's environment much like natural vision, as shown in Figure 10, which can make the operator to feel as if he is immersed in the robot's world

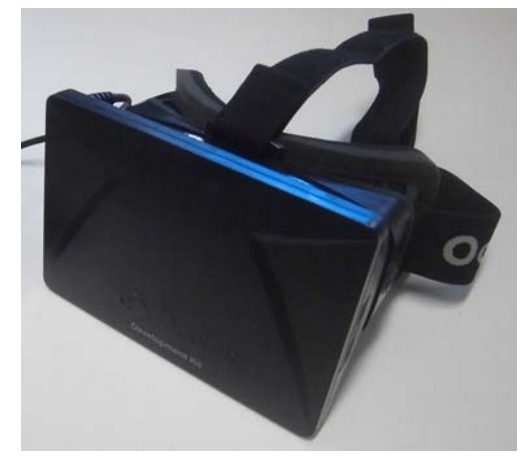

Figure 9. Oculus Rift stereo headset.

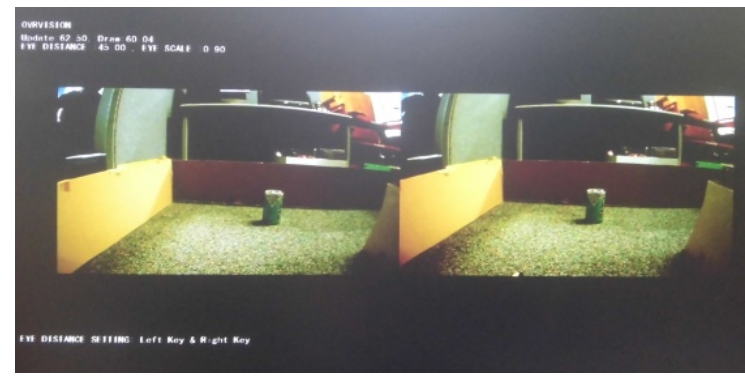

Figure 10. Stereo camera images displayed on computer screen.

\section{EXPERIMENTAL METHOD AND RESULTS}

To demonstrate the effectiveness of the proposed electrotactile feedback system at controlling a robot we setup the environment shown in Figure 11a and 11b. The aim of the experiment is to drive the mobile robot as quickly as possible through the environment and relocate the cans, one by one, from position A to position $\mathrm{B}$.

Six subjects were asked to participate in the experiment. None of the participants had any previous experience at controlling the robot or exposure to electro-tactile feedback. Prior to commencement of the trial, each operator was explained the task and fitted with the data glove and asked to adjust the maximum and minimum level of intensity of the electro-tactile feedback signals to suit their preferences.

Maximum TENS stimulus on a finger indicates that the corresponding sensor has detected an object within $3 \mathrm{~cm}$ of the sensor. Sensor range readings of $25 \mathrm{~cm}$ or greater produced the minimum TENS stimulus. Hence, the main purpose of the electro-tactile feedback is to improve the operator's awareness of the environment and to facilitate avoiding collisions.
To gauge the benefit of having electro-tactile feedback each subject was asked to perform the required task twice. Three subjects were asked to first perform the task with the electro-tactile feedback turned off and then repeat the task with the electro-tactile feedback turned on. The other three subjects were asked to do the opposite, namely, first perform the task with the electro-tactile-feedback turned on and then repeat the task with the electro-tactile feedback turned off.

All subjects were able to complete the required task considerably faster with the electro-tactile feedback enabled and with less collisions. The improvement due to electrotactile feedback ranged from between $25 \%$ to $50 \%$ in speed and $35 \%$ to $75 \%$ for collisions. There was no significant difference between the two groups. All participants reported that the experience felt as if they were inside the robot and that their right hand felt as if it was part of the robot's body when the electro-tactile feedback was enabled.
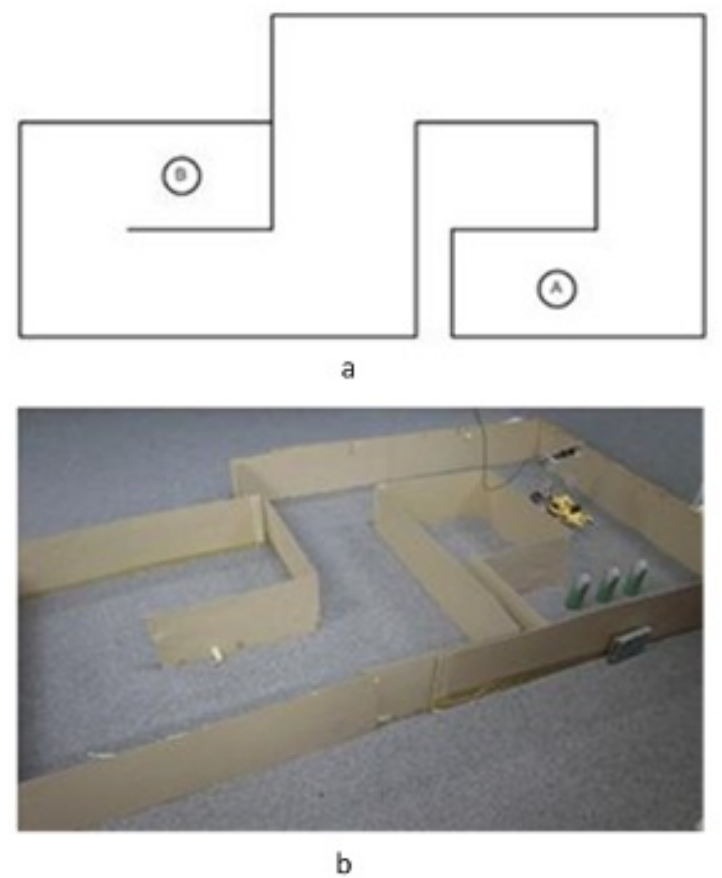

Figure 11. Obstacle avoidance obstacles experiment setup.

\section{CONCLUSION}

This paper presents an immersive tele-operation system involving remote 3D stereo vision, electro-tactile feedback and hand gesture based control. This feedback system is relative inexpensive to devise, easy to setup, versatile and avoids complicated mechanical hardware required by most other tactile feedback systems. The experimental results show how this system can facilitate control of a mobile robot and achieve some degree of embodiment within the robot and tele-presence within the robot's environment. 


\section{REFERENCES}

[1] Sheridan, T.B., Telerobotics. Automatica, 1989. 25(4): p. 487-507.

[2] Sungsik, H., Unghui, L., Hyunchul, S., Jong-Beom, P,. Jong Ho, N. Development of an unmanned coal mining robot and a tele-operation system. in Control, Automation and Systems (ICCAS), 2011 11th International Conference on. 2011.

[3] Boyle, B.G., R. McMaster, and J. Mixon. Teleoperation of an underwater robotic repair system using graphical simulation. in Control of Remotely Operated Systems: Teleassistance and Telepresence, IEE Colloquium on. 1995.

[4] Kron, A., Schmit, G., Petzold, B., Zah, M.I., Hinterseer, P., Steinbach, E. Disposal of explosive ordnances by use of a bimanual haptic telepresence system. in Robotics and Automation, 2004. Proceedings. ICRA '04. 2004 IEEE International Conference on. 2004.

[5] S. Hirche, B.S., and M. Buss, Transparent exploration of remote environments by internet telepresence. Proc. Int. Workshop HighFidelity Telepresence Teleaction/IEEE Conf/ HUMANOIDS, 2003.

[6] Sheridan, T.B., Space teleoperation through time delay: review and prognosis. Robotics and Automation, IEEE Transactions on, 1993. 9(5): p. 592-606.

[7] King, C.H., et al., Tactile Feedback Induces Reduced Grasping Force in Robot-Assisted Surgery. Haptics, IEEE Transactions on, 2009. 2(2): p. 103-110.

[8] Dolezal, L., The Remote Body: The Phenomenology of Telepresence and Re-Embodiment. Human Technology. Human Technology, 2009. 5: p. Dolezal, Luna.

[9] Haans, A. and W.A. Ijsselsteijn, Embodiment and telepresence: Toward a comprehensive theoretical framework. Interacting with Computers, 2012. 24(4): p. 211-218.

[10] Blanke, O. and T. Metzinger, Full-body illusions and minimal phenomenal selfhood. Trends in Cognitive Sciences, 2009. 13(1): p. 713.

[11] Biocca, F. The cyborg's dilemma: embodiment in virtual environments. in Cognitive Technology, 1997. Humanizing the Information Age. Proceedings. Second International Conference on. 1997.

[12] Kilten, K., R. Groten, and M. Slater, The Sense of Embodiment in Virtual Reality. Presence: Teleoperators \& Virtual Environments, 2012. 21(4): p. 373-387.

[13] Lenggenhager, B., S.T. Smith, and O. Blanke, Functional and Neural Mechanisms of Embodiment: Importance of the Vestibular System and the Temporal Parietal Junction, in Reviews in the Neurosciences. 2006. p. 643.

[14] Tsakiris, M., G. Prabhu, and P. Haggard, Having a body versus moving your body: How agency structures body-ownership. Consciousness and Cognition, 2006. 15(2): p. 423-432.

[15] Freeman, J., Bouwhuis, DG., IJsselsteijn, WA., Hamberg, R., Ridder, de, H. Perceived depth and the feeling of presence in 3DTV. Displays, 1998. 18(4): p. 207-214.
[16] Pamungkas, D.S. and K. Ward. Tele-operation of a robot arm with electro tactile feedback. in Advanced Intelligent Mechatronics (AIM), 2013 IEEE/ASME International Conference on. 2013.

[17] Botvinick, M. and J. Cohen, Rubber hands 'feel' touch that eyes see. Nature, 1998. 391(6669): p. 756.

[18] Zhang, H. and J.P. Ostrowski, Visual motion planning for mobile robots. Robotics and Automation, IEEE Transactions on, 2002. 18(2): p. 199208.

[19] Kai-Tai, S. and T. Wen-Hui, Environment perception for a mobile robot using double ultrasonic sensors and a CCD camera. Industrial Electronics, IEEE Transactions on, 1996. 43(3): p. 372-379.

[20] Drascic, D., P. Milgram, and J. Grodski. Learning effects in telemanipulation with monoscopic versus stereoscopic remote viewing. in Systems, Man and Cybernetics, 1989. Conference Proceedings., IEEE International Conference on. 1989

[21] Levy, M L; Chen, J C; Moffitt, K; Corber, Z; McComb, J G. Stereoscopic head-mounted display incorporated into microsurgical procedures: technical note. Neurosurgery, 1998. 43(2): p. 392-5.

[22] Gallo, S., Santos-Carreras, L,. Rognini, G., Hara, M., Yamamoto, A.,Higuchi, T. Towards multimodal haptics for teleoperation: Design of a tactile thermal display. in Advanced Motion Control (AMC), 2012 12th IEEE International Workshop on. 2012.

[23] A.Bloomfied, I.B., Virtual Training via Vibrotactile Arrays. Presence: Teleoperators and Virutal Environments, 2008. 17: p. 103-120.

[24] Dongseok, R., Chang-Soon, H., Sungchul., K., Munsang, K., Jae-Bok, S. Wearable haptic-based multi-modal teleoperation of field mobile manipulator for explosive ordnance disposal. in Safety, Security and Rescue Robotics, Workshop, 2005 IEEE International. 2005.

[25] Kajimoto, H., Inami, M., Kawakami, N., Tachi, S. SmartTouchaugmentation of skin sensation with electrocutaneous display. in Haptic Interfaces for Virtual Environment and Teleoperator Systems, 2003. HAPTICS 2003. Proceedings. 11th Symposium on. 2003.

[26] Wildenbeest, J., Abbink, D., Heemskerk, C., van der Helm, F., Boessenkool, H. The Impact of Haptic Feedback Quality on the Performance of Teleoperated Assembly Tasks. Haptics, IEEE Transactions on, 2012. PP(99): p. 1-1.

[27] Sagardia, M.,Weber, B., Hulin, T., Preusche, C., Hirzinger, G. Evaluation of visual and force feedback in virtual assembly verifications. in Virtual Reality Short Papers and Posters (VRW), 2012 IEEE. 2012.

[28] Stanley, A.A. and K.J. Kuchenbecker, Evaluation of Tactile Feedback Methods for Wrist Rotation Guidance. Haptics, IEEE Transactions on, 2012. 5(3): p. 240-251.

[29] Ijsselsteijn, W.A., Presence in depth. 2004, Technische Universiteit Eindhoven (The Netherlands): Ann Arbor. p. 276-276 p.

[30] Peruzzini, M., Electro-Tactile Device for Texture Simulation. IEEE/ASME International Conference on Mechatronics and Embedded Systems and Applications (MESA), 2012: p. 178-183. 